\title{
Presencia del virus del papiloma humano y del virus de Epstein-Barr en biopsias de cáncer de mama como posibles factores de riesgo
}

\section{Marla Ladera ${ }^{1, *}$, Andreina Fernandes ${ }^{1}$, Marco López $^{2}$, Adriana Pesci-Feltri $^{2}$, Maira Ávila y María Correnti ${ }^{1}$}

${ }^{1}$ Laboratorio de Genética Molecular, Instituto de Oncología y Hematología, Ministerio del Poder Popular para la Salud (MPPS);

${ }^{2}$ Servicio de Ginecología, Unidad de Patología Mamaria, Hospital Universitario de Caracas, MPPS, Caracas, Venezuela

Recibido el 8 de octubre de 2016; aceptado el 25 de febrero de 2017

Disponible en Internet el 4 de agosto de 2017

\section{PALABRAS CLAVE \\ Cáncer de mama; \\ Virus del papiloma \\ humano; \\ Virus de Epstein-Barr; \\ Factores de riesgo}

\begin{abstract}
Resumen Introducción: El cáncer de mama en Venezuela representa la primera causa de muerte oncológica en la población femenina. Se han reportado muchos factores de riesgo que favorecen su aparición; sin embargo, en las últimas décadas el virus del papiloma humano (VPH) y el virus de Epstein-Barr (VEB) se han asociado como posibles factores de riesgo en el desarrollo de esta malignidad. Objetivo: Detectar la presencia de VPH y VEB en biopsias de cáncer de mama, para establecer una posible relación entre la infección por estos agentes vi-g rales y el desarrollo de esta patología. Métodos: Se recolectaron biopsias frescas de pacientes con cáncer de mama y de pacientes con patología benigna de la mama, que asistieron a cirugia en el Hospital Universitario de Caracas. Se realizaron la detección y la tipificación de VPH mediante el estuche comercial INNOLIPA ${ }^{\circledR}$ HPV Genotyping Extra (Innogenetics), y se detectó el genoma del VEB mediante el estuche comercial Epstein-Barr virus BMLF1 (Maxim Biotech, Inc.). Resultados: El 63,6 y el 13,6\% de los casos de cáncer de mama fueron positivos para ADN de VPH y VEB, respectivamente, mientras que las muestras de patología benigna presentaron un $4,5 \%$ de positividad para cada uno de los virus. El $42,9 \%$ de las muestras de cáncer de mama presentó infección mixta con genotipos de alto y bajo riesgo oncogénico de VPH. Conclusión: Podemos sugerir que el VPH y el VEB representan importantes factores de riesgo para el cáncer de mama, pero son necesarios estudios que permitan dilucidar mejor el papel de estos virus en el desarrollo de la enfermedad. (creativecommons.org/licenses/by-nc-nd/4.0/).
\end{abstract}

${ }^{*}$ E-mail para correspondencia: marla.ladera92@gmail.com (M. Ladera) 


\section{INTRODUCCIÓN}

El cáncer de mama es el segundo cáncer más frecuente en el mundo. La Agencia Internacional para la Investigación del Cáncer, en el año 2012, estimó 1,6 millones de casos nuevos de cáncer de mama, representando el $25 \%$ de todos los tipos de cáncer diagnosticados en ese año'. En Venezuela, según el Anuario de Mortalidad del Ministerio del Poder Popular para la Salud, en 2012 el cáncer de mama fue la primera causa de muerte oncológica en la población femenina, reportándose 2067 muertes y convirtiéndose esta patología en un alarmante problema de salud pública, que si bien puede afectar a los hombres, las cifras vigentes de mortalidad masculina en el país no superan los 10 casos anuales².

Las probabilidades de desarrollar cáncer de mama aumentan o disminuyen según el número de factores de riesgo que se conjugan, entre los cuales se incluyen el ambiente, los genes y el estilo de vida. Actualmente se conocen bien varios factores de riesgo asociados con el cáncer de mama, pero en la mayoría de las mujeres afectadas no es posible identificar ningún factor en particular relacionado con el desarrollo de la enfermedad ${ }^{3}$. En las últimas décadas se ha considerado como factor importante en el desarrollo de cáncer la acción de un grupo de virus que provocan tumores, tanto en modelos experimentales como en humanos ${ }^{4,5}$. El virus del papiloma humano (VPH) es el agente etiológico del cáncer de cuello uterino, y el virus de Epstein-Barr (VEB) está asociado principalmente con el carcinoma nasofaríngeo y el linfoma de Burkitt. Estos dos virus han sido relacionados a menudo con el desarrollo de cáncer de mama, gracias al papel oncogénico de los mismos, bien sea por la expresión de genes virales similares a genes celulares o por la expresión de proteínas con capacidad de alterar el ciclo celular ${ }^{6-9}$.

El VPH es un virus de transmisión sexual, miembro de la familia Papillomaviridae, cuyo genoma se compone de ADN bicatenario circular de aproximadamente $8000 \mathrm{pb}$. Este virus tiene especial afinidad por las células epiteliales, infectando las células de la membrana basal del epitelio escamoso estratificado del cuello uterino; sin embargo, la expresión y la replicación de sus genes dependen de la diferenciación normal del epitelio. Esta dependencia del virus de la célula hospedera, junto a la expresión de oncoproteínas virales, conduce a la célula a un estado proliferativo sin que ocurra lisis celular. Las oncoproteínas E6 y E7 son capaces de interactuar con las proteínas celulares p53 y pRb, respectivamente, afectando sus funciones en la regulación del ciclo celular y conduciendo a la inmortalización celular permanente ${ }^{7,8}$. Además, la integración del genoma del VPH interrumpe y elimina el marco abierto de lectura de E2, perdiéndose la expresión de este gen viral. De esta manera, la integración del genoma viral y la pérdida concomitante de la expresión de $E 2$ podrían ser un paso importante en el proceso carcinogénico que resulta en la expresión alterada de los genes virales $E 6$ y $E 7$, debido a que la proteína $E 2$ funciona como control negativo de la replicación viral ${ }^{7,8}$. Basándose en esta afinidad del VPH con las células epiteliales y su importante capacidad de conducir al desarrollo de cáncer ${ }^{10}$, numerosos estudios han investigado la presencia de este virus en células epiteliales de tejido mamario, y algunos trabajos han reportado poca o ninguna evidencia ${ }^{11,12}$, mientras que otros han reportado importantes cifras de infección en pacientes con cáncer de mama ${ }^{4,13-15}$, planteando además posibles vías de infección por el VPH en el tejido mamario $^{16}$.

Por su parte, el VEB es un virus envuelto, miembro de la familia Herpesviridae, el cual está constituido por un genoma de ADN lineal de doble cadena de aproximadamente $172.000 \mathrm{pb}^{17}$. Es un virus que tiene gran afinidad por los linfocitos B y las células epiteliales de la orofaringe, que son la principal vía de infección del virus, pudiendo establecer dos tipos de ciclos replicativos, uno lítico y otro de latencia en la célula hospedera'. A pesar de que el VEB se asocia con frecuencia con neoplasias linfoides humanas en pacientes inmunosuprimidos ${ }^{18}$, diversos estudios han demostrado que también existe una posible asociación con el desarrollo de malignidad en células epiteliales, como el tejido mamario, basándose principalmente en algunas observaciones quee sustentan esta hipótesis: 1) la alta incidencia de cáncer de mama masculino en países del mediterráneo endémicos de VEB; 2) el desarrollo de linfomas asociados al VEB en las mamas; y 3) las similitudes morfológicas entre los carcino= mas medulares de mama y los carcinomas nasofaríngeos ${ }^{1}$. La evidencia más específica de la asociación del VEB con el cáncer de mama ha sido la identificación de secuencias génicas del virus dentro de tumores mamarios ${ }^{20,21}$. Por otra parte, también se ha evaluado la expresión de proteínas latentes del VEB en líneas celulares de cáncer de mama in vitro $e$ in vivo ${ }^{22}$, así como también la capacidad oncogénica de sus proteínas virales ${ }^{6}$. Sin embargo, algunos estudios no han encontrado ninguna relación entre el VEB y el cáncer de mama ${ }^{23-25}$.

Estas evidencias permiten plantear una posible relación entre el cáncer de mama y la infección, bien sea por VEB o por VPH, a partir de lo cual pueden considerarse estos virus como factores de riesgo para el desarrollo de este cáncer que causa muchas muertes en la población femenina en todo el mundo. Por ello, el objetivo de este trabajo fue evaluar la presencia de VPH y de VEB en biopsias frescas de pacientes venezolanas con cáncer de mama, como posibles factores de riesgo asociados a esta patología.

\section{MATERIAL Y MÉTODOS}

\section{Pacientes}

Se evaluó de forma prospectiva, desde abril de 2014 hasta mayo de 2015, a 44 pacientes venezolanas que asistieron a consulta en el Servicio de Ginecología, Unidad de Patologia Mamaria, del Hospital Universitario de Caracas. A todas las pacientes se las invitó a participar en el estudio, con previa información del diseño y del protocolo, y firmaron un consentimiento informado aprobado por el Comité de Bioética del hospital. La selección de las pacientes se realizó bajo los siguientes criterios: pacientes con diagnóstico de carcinoma mamario en estadios 0 , I o II, pacientes con diagnóstico de carcinoma mamario en estadios III y IV que por motivos intrínsecos no recibieron terapia neoadyuvante, y pacientes con diagnóstico de patología mamaria benigna. Se excluyeron aquellas pacientes con diagnóstico de enfermedad autoinmune u otro tipo de cáncer no relacionado con el tumor primario, embarazadas, que hubieran recibido terapia neoadyuvante o que no aceptaran participar en el estudio. 
Tabla 1. Características clínicas de las pacientes con diagnóstico de cáncer de mama y patología benigna

\begin{tabular}{lcc}
\hline & Cáncer de mama & Patología benigna \\
\hline Promedio de edad & 58 años (rango: 30-86) & 29,4 años (rango: 14-50) \\
Promedio de menarquia & 12,5 años (rango: 9-17) & 12,5 años (rango: 9-17) \\
Promedio de embarazos & 3,14 embarazos (rango: 0-9) & 1,32 embarazos (rango: 0-6) \\
Promedio de parejas & 1,85 parejas (rango: 1-4) & 1,73 parejas (rango: 0-5) \\
Uso de anticonceptivos orales & $45,5 \%$ & $72,7 \%$ \\
Presencia de VPH & $63,6 \%$ & $4,5 \%$ \\
Presencia de VEB & $13,6 \%$ & $4,5 \%$ \\
\hline VEB: Virus Epstein-Barr; VPH: Virus del papiloma humano. & & \multirow{2}{0}{} \\
\end{tabular}

\section{Extracción y evaluación de la calidad del material genético}

El aislamiento del material genético fue realizado con el estuche comercial Pure Link ${ }^{T M}$ Genomic DNA Kit, de Invitrogen, siguiendo las especificaciones de la casa comercial. La evaluación de la calidad del ADN de todas las muestras se llevó a cabo según el protocolo de BIOMED-2, de Van Dongen, et $\mathrm{al}^{26}$. La mezcla de reacción se preparó utilizando $0,1 \mu \mathrm{l}$ de DNTP (100 mM), $2 \mu \mathrm{l}$ de cada iniciador (100 pM), 6,5 $\mu \mathrm{l}$ de buffer 10X, $2 \mu \mathrm{l}$ de $\mathrm{MgCl}_{2}(50 \mathrm{mM}), 0,6 \mu \mathrm{l}$ de Taq polimerasa y $28,8 \mu \mathrm{l}$ de $\mathrm{H}_{2} \mathrm{O}$ libre de nucleasas, para un volumen final de $50 \mu \mathrm{l}$. Las condiciones de amplificación fueron 7 minutos a $95{ }^{\circ} \mathrm{C}, 35$ ciclos de 30 segundos a $45{ }^{\circ} \mathrm{C}, 40$ segundos a $60{ }^{\circ} \mathrm{C}$ y 40 segundos a $70{ }^{\circ} \mathrm{C}$, y una amplificación final de 15 segundos a $70{ }^{\circ} \mathrm{C}$.

\section{Detección y tipificación del genoma del virus del papiloma humano}

La detección y la genotipificación de los 28 genotipos del $\mathrm{VPH}$, a partir de las biopsias de mama, fue llevada a cabo con el estuche INNO-LIPA ${ }^{\circledR}$ HPV Genotyping Extra (Innogenetics), siguiendo las recomendaciones de la casa comercial. Esta prueba consiste en un ensayo con sondas inmovilizadas en tiras de nitrocelulosa, basado en un principio de hibridación reversa, diseñado para la identificación de 28 genotipos diferentes del VPH mediante la detección de secuencias específicas de la región L1 del genoma del VPH. Este ensayo usa un conjunto de iniciadores SPF10 para una mayor sensibilidad de amplificación de la mayoría de los genotipos del VPH clínicamente más relevantes.

\section{Detección del genoma del virus de Epstein-Barr}

La detección del VEB se realizó con el estuche comercial Epstein-Barr virus BMLF1 de Maxim Biotech, Inc., siguiendo las especificaciones de la casa comercial. Las condiciones de la mezcla de reacción fueron las siguientes: se utilizaron $40 \mu \mathrm{l}$ de master mix (buffer, dNTP, $\mathrm{MgCl}_{2}$ ), 0,2 $\mu \mathrm{l}$ de Taq polimerasa y $10 \mu \mathrm{l}$ de la muestra de $A D N$, para obtener un volumen final de 50,2 $\mu \mathrm{l}$. Las condiciones de amplificación fueron 1 minuto a $96{ }^{\circ} \mathrm{C}, 35$ ciclos de 1 minuto a $94{ }^{\circ} \mathrm{C}, 1$ minuto a $58{ }^{\circ} \mathrm{C}, 1$ minuto a $72{ }^{\circ} \mathrm{C}$ y una amplificación final de
10 minutos a $72{ }^{\circ} \mathrm{C}$. Se consideraron positivas solo aquellas muestras en las que se observó una banda de 265 pb.

\section{Electroforesis en geles de agarosa}

Los productos de amplificación de la reacción en cadena de la polimerasa (PCR) se visualizaron mediante electroforesis en gel de agarosa al 2\%, con buffer TBE1X stock de 10X de Invitrogen (1.0 mM Tris base, $0.9 \mathrm{mM}$ borato y $0.01 \mathrm{mM}$ EDTA, $\mathrm{pH} 8.0$ ), y se tiñeron con SYBR ${ }^{\circledR}$ Safe (Invitrogen). El registro fotográfico se realizó con un fotodocumentador ChemiDoc de BioRad.

\section{Análisis estadístico}

Se realizaron análisis descriptivos, utilizando medidas de tendencia central y de dispersión (media, mediana y desviación típica) en el caso de las variables continuas, y análisis de frecuencia y tablas de contingencia en el caso de las variables discretas. Se realizó una prueba de ji al cuadrado para evaluar la dependencia de las variables, con el programa Excel de Microsoft Office 2010. Valores de $p \leq 0.05$ fueron considerados estadísticamente significativos.

\section{RESULTADOS}

En la tabla 1 se muestran las características clínicas de la población estudiada, indicando el promedio de edad, el pro-o medio de la menarquia, el promedio de embarazos a término, el promedio del número de parejas y el uso de anticonceptivos orales. Del total de muestras de cáncer de mama, el 63,6\% correspondió a pacientes con tumores en estadio II, seguidos del estadio I con un $18,2 \%$. En cuanto al diagnóstico histopatológico, los tumores de mama recolectados fueron del tipo carcinoma ductal in situ, carcinoma ductal infiltrante, carcinoma lobulillar infiltrante y carcinoma papilar infiltrante, siendo el carcinoma ductal infiltrante el que se presentó con mayor frecuencia, con $68,2 \%$.

Luego de realizada la extracción del material genético de las biopsias del estudio, se pudo corroborar la calidad del mismo mediante la identificación de cinco genes de expresión constitutiva, para garantizar que el genoma se encontrara en óptimas condiciones. Todas las muestras amplificaron los genes diana, debido a que las muestras recolectadas fueron biopsias frescas conservadas a $-80{ }^{\circ} \mathrm{C}$, sin necesidad 


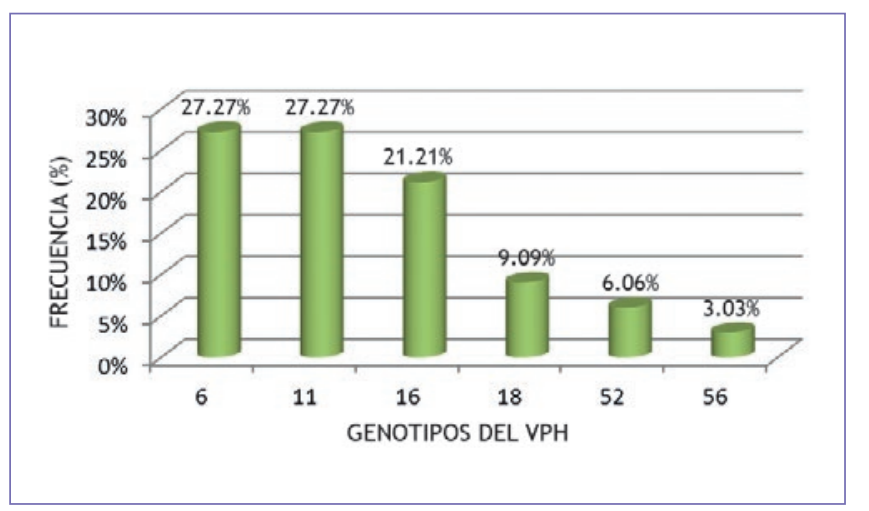

Figura 1. Frecuencia de los genotipos del VPH en las biopsias tumorales de las pacientes con carcinoma mamario.

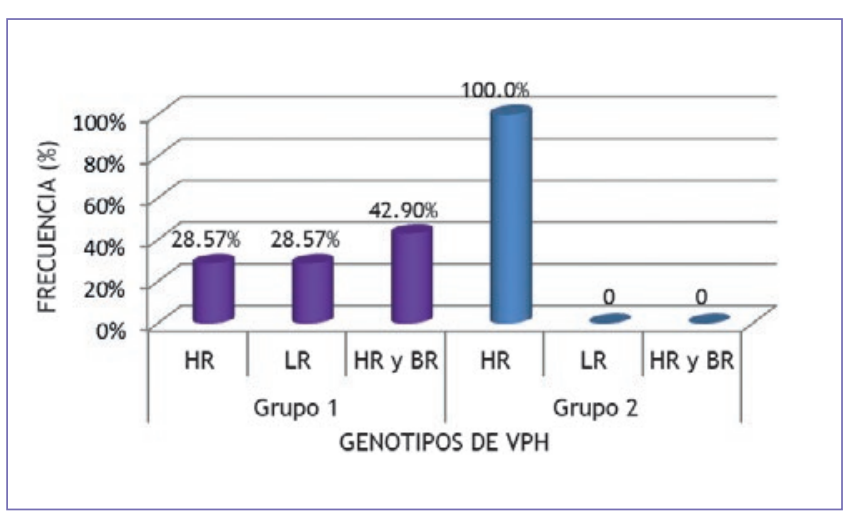

Figura 2. Frecuencia de infección por genotipos de alto y bajo riesgo encontrada en ambos grupos de estudio. Grupo 1: pacientes con cáncer de mama; grupo 2: pacientes con patología benigna. AR: alto riesgo oncogénico; BR: bajo riesgo oncogénico; AR y BR: coinfección por ambos genotipos virales.

de estar embebidas en parafina, evitando así la posible fragmentación del ADN durante el procesamiento de la muestra para preparar los bloques de parafina, lo que dificulta la detección del ADN viral.

Seguidamente se realizaron la detección y la genotipificación, y se obtuvieron 14 de 22 muestras de cáncer de mama positivas para ADN del VPH, representando el $63.6 \%$, mientras que en las muestras de tejido sano solo se detectó ADN del VPH en una muestra, representado el $4.5 \%$ de todas las pacientes con patología benigna evaluadas (Tabla 1).

Al realizar la tipificación encontramos que, en las 14 muestras positivas, los tipos más frecuentes fueron el $6 \mathrm{y}$ el 11 , con un $27.27 \%$ cada uno, seguidos por el tipo 16 con un $21.21 \%$. En cuanto a la paciente con patología benigna infectada por el VPH, se identificó el genotipo viral de alto riesgo oncogénico VPH-33. En la figura 1 se muestra un gráfico de barras con la frecuencia de cada uno de los genotipos del VPH encontrados en las pacientes con cáncer de mama.

Además, siguiendo la clasificación del VPH según el genotipo viral reportado, en este trabajo se agruparon los tipos de VPH de acuerdo con su riesgo oncogénico asociado al desarrollo de varios tipos de carcinomas. En la figura 2 se observa una representación gráfica de la frecuencia de infección por los genotipos de alto y bajo riesgo, encontrados en ambos grupos de estudio. Se halló que el $28.57 \%$ de las pacientes con cáncer de mama presentó infección úni- ca por genotipos de alto y bajo riesgo, respectivamente, y el $42.90 \%$ de las pacientes presentó coinfección por ambos grupos de genotipos. En cuanto a la muestra de tejido sano positiva para ADN del VPH, se encontró que era de alto riesgo oncogénico.

Dentro del grupo de las pacientes con cáncer, se detectó la presencia de genoma del VEB en tres de las 22 muestras evaluadas, representando un $13.6 \%$ de infección, y en las muestras de tejido sano se detectó el genoma en una mues? tra, representado el $4.5 \%$ del total (Tabla 1 ).

De las pacientes con cáncer de mama, tres mostraron co infección por VPH y VEB, correspondiendo al $13.6 \%$ de todas las pacientes evaluadas. En el grupo de patología benigna, se encontró que la única muestra positiva para ADN del VEB también era positiva para ADN del VPH, correspondiendo a una paciente con diagnóstico de mastitis abscesada. Sin embargo, no se encontró relación estadísticamente significativa entre la infección por VPH y VEB en las muestras de cáncer de mama evaluadas $(p=0.344)$.

\section{DISCUSIÓN}

Los datos clínicos de las pacientes con cáncer de mama evaluadas se corresponden con los de publicaciones previas, en las que se ha reportado que el rango de edad de las pacien tes afectadas por cáncer de mama se encuentra entre los 45 y los 65 años en Venezuela, y entre los 50 y los 80 años en países de Europa y Norteamérica, mientras que las patologías benignas están asociadas a edades tempranas de la mujer en todo el mundo ${ }^{27,28}$. Se ha planteado que la alta in cidencia de lesiones benignas en mujeres jóvenes se debe a que la carga hormonal es mayor, y el estímulo hormonal du rante el ciclo reproductivo femenino conduce a un aumento de la actividad mitótica celular; aunado a esto, si las pa cientes jóvenes presentan desequilibrio hormonal, aumenta el riesgo de desarrollar patologías benignas en este sitio. En las pacientes de edad media y tercera edad, la presencia de patologías benignas es menor proporcionalmente con el mayor número de embarazos a término, lo que es posible que vaya disminuyendo la carga hormonal ${ }^{27,28}$.

En este estudio, la presencia de VPH fue detectada en el $63.6 \%$ de las muestras de cáncer de mama, mientras que solo el $4.5 \%$ de las biopsias de tejido con patología benigna fueron positivas para este virus. Esta frecuencia de detec-o ción se encuentra dentro del rango publicado previamente en todo el mundo, con una prevalencia que oscila entre el 10 y el 86\%4,5,14,16,29-32. Akil, et al.4, en 2008, y Antonsson, et al. ${ }^{30}$, en 2011 , detectaron mediante PCR múltiple y PCR consenso un 61.06 y un $50 \%$ de casos positivos para ADN de $\mathrm{VPH}$, respectivamente.

En Latinoamérica, la tasa de infección por VPH en pacientes con cáncer de mama es baja; se ha reportado un $8-40 \%$ de positividad ${ }^{14,15,31-33}$. Por lo tanto, nuestro trabajo es el primero en reportar una alta frecuencia de VPH en el tejido mamario maligno de pacientes venezolanas. Sin embargo, algunos estudios no han detectado VPH en tejido tumoral ni en tejido normal de la mama ${ }^{11,34}$. Esta diferencia en los reportes puede atribuirse al número de muestras evaluadas, a diferencias en la metodología y la sensibilidad de los métodos usados, tales como el uso de diferentes juegos de iniciadores, o al tipo de muestra utilizada. 
Al realizar la identificación de los genotipos del VPH encontramos que las 14 muestras positivas presentaron infecciones únicas y mixtas, con los genotipos de alto riesgo oncogénico 16, 18, 52 y 56 , y de bajo riesgo oncogénico 6 y 11. En los tumores malignos, el genotipo de alto riesgo más frecuente fue el 16 , con el $21.21 \%$, y en cuanto a los genotipos de bajo riesgo, los más encontrados fueron el 6 y el 11, con un 27.27\% cada uno. En la paciente con patología benigna infectada por el VPH se identificó el genotipo 33, de alto riesgo oncogénico. Estos genotipos identificados coinciden con los reportados en el resto del mundo, donde el genotipo de alto riesgo oncogénico más frecuentemente detectado es el 16, en un 20 a un $90 \%$ de los $\operatorname{casos}^{21,35}$.

Agrupando los genotipos virales según su riesgo oncogénico, obtuvimos que el $28.57 \%$ de las pacientes con cáncer de mama presentó infección única por genotipos de alto y bajo riesgo, respectivamente, y el $42.90 \%$ de las pacientes positivas para VPH presentó coinfección por ambos grupos de genotipos.

Los genotipos de alto riesgo 16 y 18 están asociados con un crecimiento acelerado de las células epiteliales cervicales, ya que tienden a integrarse en el genoma y conducir a la proliferación celular descontrolada debido a la inhibición de la función de las proteínas celulares p53 y pRb ${ }^{36}$. En este trabajo encontramos que, del $57.1 \%$ de las pacientes infectadas por VPH 16 o 18, el 75\% tenían reportada en la historia clínica una alta expresión del marcador de proliferación celular Ki67, mientras que en el resto de las pacientes infectadas con otros genotipos virales $(6,11,52$ y 56) solo una de seis pacientes tenía reportada sobreexpresión de Ki67, lo que sugiere que la presencia de los genotipos de alto riesgo (16 y 18) puede conducir a una proliferación celular descontrolada en el tejido mamario.

Desde que se reportó por primera vez la presencia de VEB, el número de estudios que apunta hacia el potencial oncogénico de este y otros virus, y su relación con diferentes tipos de cáncer, ha ido aumentando progresivamente. En este sentido, se han realizado nuevas investigaciones que asocian el VEB con otros tipos de linfomas y varios carcinomas, como son el carcinoma gástrico, el de pulmón y, recientemente, el mamario ${ }^{4,37,38}$. Estas asociaciones están basadas en el tipo de células que es capaz de infectar el VEB, las cuales son células B y células epiteliales. Además, la mayoría de estos tumores están caracterizados por la presencia de múltiples copias extracromosómicas de genoma viral y la expresión de genes latentes codificados por el VEB, lo cual contribuye al fenotipo maligno ${ }^{39}$.

En este estudio se detectó la presencia de VEB en el $13.6 \%$ de nuestras pacientes con cáncer de mama, mientras que en aquellas con patología benigna se detectó ADN del VEB solo en una, representando el 4.5\%. Este resultado se encuentra por debajo de los valores reportados en otros estudios realizados en el mundo, los cuales oscilan entre el 21 y el 55\% de positividad para ADN del VEB, dependiendo del tipo de técnica empleada en la detección ${ }^{4,19,40}$. En los trabajos que emplearon algún tipo de PCR, al igual que en nuestro estudio, se detectaron bajas frecuencias de infección, como en el de Xue, et al. ${ }^{41}$, quienes detectaron, mediante PCR específica de transcriptasa reversa para el gen BZLF1, el ARN viral en el $17 \%$ de los casos de cáncer de mama, o el trabajo de Yahia, et al. ${ }^{42}$, quienes detectaron un $11 \%$ de positividad para ADN del VEB en los casos de cáncer de mama mediante PCR con iniciadores específicos para el gen EBNA-1.

En Latinoamérica son muy pocos los estudios que han evaluado la presencia del VEB en pacientes con cáncer de mama, y han reportado una frecuencia de infección entre el 6 y el $31 \%^{31,43}$. Las diferencias obtenidas con respecto a otros estudios pueden deberse a la variación en la epidemiología del VEB según la región geográfica.

Por lo tanto, siendo el VEB un cofactor en el desarrollo de varias malignidades, incluyendo diferentes carcinomas (nasofaríngeo, gástrico $)^{18}$, podemos sugerir que su presencia en tumores del tejido mamario podría ser un factor de riesgo importante para el cáncer de mama, debido a su potencial oncogénico. Sin embargo, es importante tomar en consideración la carga del ADN genómico del virus y la cantidad de ADN evaluado en las muestras, para evitar las discrepancias en la detección de ADN del virus.

En cuanto a la coinfección por VPH y VEB en las pacien= tes con cáncer de mama, se observó que en las tres que presentaron ADN del VEB también se detectó ADN del VPH, correspondiendo al $13.6 \%$ de todas las pacientes con car cinoma mamario evaluadas. Esta frecuencia de coinfección es intermedia con respecto a la reportada en otros estudios internacionales, en los cuales oscila entre el 2.1 y el $38 \%^{20,34}$ Es importante destacar que son pocos los estudios que han evaluado la presencia de este virus en Sudamérica. Aguayo, et al. ${ }^{31}$, en Chile, estudiaron la coinfección por VEB y VPH, y encontraron un $6.5 \%$ de positividad para VEB en muestras de cáncer de mama.

Este es, para el mejoramiento de nuestros conocimientos, el primer estudio que evalúa la presencia de ambos virus en pacientes venezolanas con cáncer de mama. A pesar de que no hubo relación estadísticamente significativa en cuanto a la presencia de ambos agentes virales, no puede obviarse el comportamiento biológico de los virus.

\section{CONCLUSIONES}

La presencia de VPH se detectó en el $63.6 \%$ de las pacientes con cáncer de mama, mientras que el VEB fue detectado en el $13.6 \%$. A pesar de que no hubo diferencias estadísticamente significativas, podemos sugerir que el VPH y el VEB podrían actuar como importantes factores de riesgo en la patogénesis del cáncer de mama, debido a sus características oncogénicas. Nuestro hallazgo está basado principalmente en la detección del genoma viral por la técnica de PCR e hibridación reversa, por lo que harían falta pruebas confirmatorias y se necesitan más investigaciones para determinar el papel del VPH y del VEB en la etiología o la progresión del cáncer de mama, incluyendo la evaluación de la carga viral para ambos agentes y là determinación de la integración viral del VPH al genoma del hospedero, ya que se conoce que este es un paso importante en el desarrollo de la carcinogénesis, afectando importantes puntos de control de la replicación viral. Además, es preciso incrementar el número de muestras, lo que permitirá observar una tendencia más clara en cuanto a la relación entre la presencia de los virus y el desarrollo de cáncer de mama. 


\section{AGRADECIMIENTOS}

Los autores agradecen el financiamiento otorgado por los proyectos de investigación PEII n. 2012001201 y FONACIT G2005000408.

\section{CONFLICTO DE INTERESES}

Los autores declaran no tener ningún conflicto de intereses.

\section{BIBLIOGRAFÍA}

1. GLOBOCAN 2012. Fact sheets: breast cancer. Estimated incidence, mortality and prevalence worldwide in 2012. 2015. (Consultado en abril de 2015.) Disponible en: http://globocan.iarc.fr/old/FactSheets/ cancers/breast-new.asp

2. Ministerio del Poder Popular para la Salud. Anuario de mortalidad 2011 de Venezuela. Caracas, Venezuela; enero de 2014. (Consultado en septiembre de 2014.) Disponible en: http://www.bvs.gob.ve/anuario/ Anuario2011.pdf

3. Peralta O. Cáncer de mama. Epidemiología y factores de riesgo. Cuad Med Soc. 2007;47:18-30.

4. Akil N, Yasmeen A, Kassab A, et al. High-risk human papillomavirus infections in breast cancer in Syrian women and their association with Id-1 expression: a tissue microarray study. Br J Cancer. 2008;99:404-7.

5. Lawson J, Glenn W, Salmons B, et al. Mouse mammary tumor virus-like sequences in human breast cancer. Cancer Res. 2010;70:3576-85.

6. Young L, Murray P. Epstein-Barr virus and oncogenesis: from latent genes to tumours. Oncogene. 2003;22:5108-21.

7. Egawa N, Egawa K, Griffin H, et al. Human papillomaviruses; epithelial tropisms, and the development of neoplasia. Viruses. 2015;7:3863-90.

8. Ghittoni R, Accardi R, Chiocca S, et al. Role of human papillomaviruses in carcinogenesis. Ecancer. 2015;9:526.

9. De Paschale M, Clerici P. Serological diagnosis of Epstein-Barr virus infection: problems and solutions. World J Virol. 2012;1:31-43.

10. Correnti M, Medina F, Cavazza M, et al. Human papillomavirus (HPV) type distribution in cervical carcinoma, low-grade, and high-grade squamous intraepithelial lesions in Venezuelan women. Gynecol Oncol. 2011;121:527-31.

11. Hachana M, Ziadi S, Amara $\mathrm{K}$, et al. No evidence of human papillomavirus DNA in breast carcinoma in Tunisian patients. Breast. 2010;19:541-4.

12. Vernet M, Mena M, Alemany L, et al. Human papillomavirus and breast cancer: no evidence of association in a Spanish set of cases. Anticancer Res. 2015;35:851-6.

13. Widschwendter A, Brunhuber T, Wiedemair A, et al. Detection of human papillomavirus DNA in breast cancer of patients with cervical cancer history. Clin Diagn Virol. 2004;31:292-7.

14. Fernandes A, Pesci-Feltri A, García I, et al. Evaluación de la infección por virus de papiloma humano en pacientes con cáncer de mama. Rev Venez Oncol. 2015;27:22-9.

15. Fernandes A, Bianchi G, Pesci-Feltri A, et al. Presence of human papillomavirus in breast cancer and its association with prognostic factors. Ecancer. 2015;9:548.

16. De Villiers E, Sandstrom R, zurHausen H, et al. Presence of papillomavirus sequences in condylomatous lesions of the mamillae and in invasive carcinoma of the breast. Breast Cancer Res. 2005;7:1-11.

17. Tao Q, Young L, Woodman C, et al. Epstein-Barr virus (EBV) and its associated human cancers - genetics, epigenetics, pathobiology and novel therapeutics. Front Biosci. 2006;11:2672-713.

18. Rickinson A. Co-infections, inflammation and oncogenesis: future directions for EBV research. Semin Cancer Biol. 2014;26:99-115.

19. Joshi D, Quadri M, Gangane N, et al. Association of Epstein Barr virus infection (EBV) with breast cancer in rural Indian women. Ploss One. 2009;4:8.
20. Glenn W, Heng B, Delprado W, et al. Epstein-Barr virus, human papillomavirus and mouse mammary tumor virus as multiple viruses in breast cancer. Plos One. 2012;7:10.

21. Al Moustafa A, Al-Antary N, Aboulkassim T, et al. Co-prevalence of Epstein-Barr virus and high-risk human papillomaviruses in Syrian women with breast cancer. Hum Vaccin Immunother. 2016;12:1936-9.

22. Arbach H, Viglasky V, Lefeu F, et al. Epstein-Barr virus (EBV) genome and expression in breast cancer tissue: effect of EBV infection of breast cancer cells on resistance to paclitaxel (taxol). J Virol. 2006;80:845-53.

23. Kadivar M, Monabati A, Joulace A, et al. Epstein-Barr virus and breast cancer: lack of evidence for an association in Iranian women. Pathol Oncol Res. 2011;17:489-92.

24. Fadavi $P$, Rostamian $M$, Arashkia A, et al. Epstein-Barr virus may not be associated with breast cancer in Iranian patients. Oncol Discov 2013;1:10

25. Fu L, Wang D, Shah W, et al. Association of human papillomavirus type 58 with breast cancer in Shaanxi province of China. J Med Virol? 2015;87:1034-40.

26. Van Dongen J, Langerak A, Brüggemann $M$, et al. Design and standar dization of PCR primers and protocols for detection of clonal immunoglobulin and T-cell receptor gene recombinations in suspect lymphoproliferations: report of the BIOMED-2 concerted action BMH4-CT98-3936. Leukemia. 2003; 7:2257-317.

27. Hómez B. Hormonas en la mama: de la fisiología a la enfermedad. Revisión. Rev Venez Endocrinol Metab. 2008;6:9-14.

28. Hernández D, Borges R, Márquez G, et al. Factores de riesgo conocidos para cáncer de mama. Rev Venez Oncol. 2010;22:16-31.

29. Cantú de León D, Pérez D, Nemcova J, et al. Human papillomavirus (HPV) in breast tumors: prevalence in a group of Mexican patients. BMC Cancer. 2009;9:26.

30. Antonsson A, Spurr T, Chen A, et al. High prevalence of human papillomaviruses in fresh frozen breast cancer samples. J Med Virol. 2011;83:2157-63.

31. Aguayo F, Khan N, Koriyama C, et al. Human papillomavirus and Epstein-Barr virus infections in breast cancer from Chile. Infect Agents Cancer. 2011;6:7.

32. Herrera R, Vela T, Carrillo A, et al. High-risk human papillomavirus (HPV) DNA sequences in metaplastic breast carcinomas of Mexican women. BMC Cancer. 2013;13:445.

33. Pereira A, Lorenzetti M, González R, et al. Presence of human papilloma virus in a series of breast carcinoma from Argentina. Plos One. 2013;8:1-4.

34. Chang $\mathrm{P}$, Wang $\mathrm{T}$, Yao $\mathrm{Q}$, et al. Absence of human papillomavirus in patients with breast cancer in north-west China. Med Oncol. 2012;29:521-5.

35. Khan N, Castillo A, Koriyama C, et al. Human papillomavirus detected in female breast carcinomas in Japan. Br J Cancer. 2008;99:408-14.

36. Rivera R, Delgado J, Painel V, et al. Mecanismo de infección y transformación neoplásica producido por virus papiloma humano en ef epitelio cervical. Rev Chil Obstet Ginecol. 2006;71:135-40.

37. Ryan J, Jones R, Kenney S, et al. Epstein-Barr virus-specific methylation of human genes in gastric cancer cells. Infect Agent Cancer. 2010;5:27.

38. Mazouni C, Fina F, Romain S, et al. Outcome of Epstein-Barr virus-associated primary breast cancer. Mol Clin Oncol. 2015;3:295-8.

39. Lorenzetti M, De Matteo E, Gass H, et al. Characterization of Epstein Barr virus latency pattern in Argentine breast carcinoma. Plos One. 2010;5:1-7.

40. Aboulkassim T, Yasmeen A, Akil N, et al. Incidence of Epstein-Barr virus in Syrian women with breast cancer: a tissue microarray study. Hum Vaccin Immunother. 2015;11:951-5.

41. Xue S, Lampert I, Haldane J, et al. Epstein-Barr virus gene expression in human breast cancer: protagonist or passenger? $\mathrm{Br} \mathrm{J}$ Cancer: 2003;89:113-9

42. Yahia Z, Adam A, Elgizouli M, et al. Epstein Barr virus: a prime candi $=$ date of breast cancer aetiology in Sudanese patients. Infect Agent Cancer. 2014;9:9.

43. Preciado $M$, Chabay $P$, Actis A, et al. Epstein-Barr virus in breast carcinoma in Argentina. Arch Pathol Lab Med. 2005;129:371-81. 